\title{
Dispersion of a single hole in the $t-J$ model
}

\author{
T. K. Lee \\ Department of Physics, Virginia Tech, Blacksburg, Virginia 24060 and Institute of Physics, Academia Sinica, Nankang, Taiwan \\ C. T. Shih \\ Department of Physics, National Tsing Hua University, Hsinchu, Taiwan
}

(Received 9 April 1996; revised manuscript received 27 August 1996)

\begin{abstract}
The dispersion of a single hole in the $t-J$ model obtained by the exact result of 32 sites and the results obtained by self-consistent Born approximation and the Green function Monte Carlo method can be simply derived by a mean-field theory with $d$-wave resonating-valence-bond ( $d$-RVB) and antiferromagnetic order parameters. In addition, it offers a simple explanation for the difference observed between those results. The presence of the extended van Hove region at $(\pi, 0)$ is a consequence of the $d$-RVB pairing instead of the antiferromagnetic order. Results including $t^{\prime}$ and $t^{\prime \prime}$ are also presented and explained consistently in a similar way. [S0163-1829(97)08701-8]
\end{abstract}

Important advances in the study of high-temperature superconductors have been made recently by angle-resolved photoemission (ARPES) experiments. ${ }^{1,2}$ Extended van Hove singularity (EVHS) near the Fermi surface of the superconductors is found. In particular, Wells et al. ${ }^{3}$ have measured the ARPES for the insulating $\mathrm{Sr}_{2} \mathrm{CuO}_{2} \mathrm{Cl}_{2}$ and find good agreement with the prediction of the $t-J$ model about the bandwidth and other features. Although there is substantial disagreement about the position of the energy level near $(\pi, 0)$.

There were many theoretical studies ${ }^{4}$ of the properties of a single hole in a quantum antiferromagnet. The theoretical data used in comparison with ARPES data are obtained from the $t-J$ model by using the self-consistent Born approximation $(\mathrm{SCBA})^{5-7}$ to treat the scattering of a single hole with the spin waves of the Néel state. The result of SCBA shows an EVHS near $(\pi, 0)$. The SCBA dispersion of the single hole is also reproduced in the Green function Monte Carlo (GFMC) approach by Dagotto et al. ${ }^{8}$ Based on these results, Dagotto and collaborators ${ }^{9}$ have argued that the EVHS is due to the antiferromagnetism and have proposed the antiferromagnetic van Hove theory to explain the mechanism of superconductivity.

Recently Leung and Gooding ${ }^{10}$ have solved the spectra exactly for a single hole in a 32 -site square cluster. The dispersion relation obtained is very similar to the result of SCBA except they have not observed the exact symmetry resulted from folding the Brillouin zone (BZ) in half due to the antiferromagnetic long-range order. It should be noticed that this asymmetry is also observed in the GFMC result ${ }^{8}$ for a $12 \times 12$ cluster.

In this paper we will show that the results obtained by SCBA (Ref. 7) and GFMC (Ref. 8) as well as exact diagonalization ${ }^{10}$ can be easily reproduced by a mean-field theory including both $d$-wave resonating-valence-bond $(d$-RVB $)$ and antiferromagnetic order parameters. The presence of the EVHS is associated with the $d$-RVB pairing.

We have also looked at a more general $t-J$ model by including next-nearest-neighbor hopping, $t^{\prime}$, and next-nextnearest-neighbor hopping, $t^{\prime \prime}$. It is shown below that the complicated dispersions obtained by including $t^{\prime}$ and $t^{\prime \prime}$ are easily understood in terms of the mean-field theory. A better agreement with the ARPES of the insulating $\mathrm{Sr}_{2} \mathrm{CuO}_{2} \mathrm{Cl}_{2}$ (Ref. 3) could be obtained by tuning the values of $t^{\prime}$ and $t^{\prime \prime},{ }^{11,12}$ but there is still discrepancy.

Several years ago a number of groups ${ }^{13-15}$ used the variational Monte Carlo method to study the phase diagram of the $t-J$ model at small hole density. The antiferromagnetic phase boundary obtained agrees fairly well with experiments. ${ }^{13}$ Near half filling the best trial function has antiferromagnetism and $d$-wave resonating-valence-bond ( $d$-RVB) (Ref. 16) coexist. But no analytical mean-field theory was discussed.

The two order parameters, Néel order and singlet $d$-RVB pairing, can be considered simultaneously in a meanfield theory of the $t-J$ model, which is given by

$$
H=-t \sum_{\langle i, j\rangle \sigma}\left(\widetilde{c_{i \sigma}} \widetilde{c_{j \sigma}}+\text { H.c. }\right)+J \sum_{\langle i, j\rangle}\left(\mathbf{S}_{i} \cdot \mathbf{S}_{j}-\frac{1}{4} n_{i} n_{j}\right),
$$

where $\langle i, j\rangle$ is the nearest-neighbor pairs and $\widetilde{c_{i \sigma}}$ $=c_{i \sigma}\left(1-n_{i,-\sigma}\right)$. At half filling, we shall only consider the $\mathbf{S}_{i} \cdot \mathbf{S}_{j}$ term which involves three mean-field order parameters. The staggered magnetization is $m_{s}=\left\langle S_{A}^{z}\right\rangle=-\left\langle S_{B}^{z}\right\rangle$, where the lattice is divided into $A$ and $B$ sublattices. The uniform bond-order parameter is $\chi=\left\langle\Sigma_{\sigma} c_{i \sigma}^{+} c_{j \sigma}\right\rangle$, and $d$-RVB is $\Delta=\left\langle c_{j \downarrow} c_{i \uparrow}-c_{j \uparrow} c_{i \downarrow}\right\rangle$ if $i$ and $j$ are nearest-neighbor sites in $x$ direction and $-\Delta$ for $y$ direction. The mean-field Hamiltonian can be diagonalized and this is done recently by Inaba et al. ${ }^{17}$ in the slave-boson formalism. They have examined the mean-field phase diagram of these order parameters. We shall adopt a slightly different approach.

Instead of taking into account all three order parameters together in the mean-field Hamiltonian, we first consider the staggered magnetization $m_{s}$ and uniform bond order $\chi$. They produce upper and lower spin-density-wave (SDW) bands with dispersions: $\pm \xi_{k}= \pm\left[\epsilon_{k}^{2}+\left(J m_{s}\right)^{2}\right]^{1 / 2}$ where $\epsilon_{k}=-\frac{3}{4}$ 
$J \chi\left(\cos k_{x}+\cos k_{y}\right)$. The states in these bands are then paired. At half filling the mean-field Hamiltonian, in addition to a constant, is of the form

$$
\begin{aligned}
H_{\mathrm{MF}}= & \sum_{\mathbf{k}, \sigma}-\xi_{k} a_{\mathbf{k} \sigma}^{+} a_{\mathbf{k} \sigma}+\sum_{\mathbf{k}, \sigma} \xi_{k} b_{\mathbf{k} \sigma}^{+} b_{\mathbf{k} \sigma} \\
& +\sum_{\mathbf{k}} \Delta_{k}\left(a_{-\mathbf{k} \uparrow} a_{\mathbf{k} \downarrow}-b_{-\mathbf{k} \uparrow} b_{\mathbf{k} \downarrow}\right)+\text { H.c., }
\end{aligned}
$$

where $\Delta_{k}=\frac{3}{4} J \Delta d_{k}$, and $d_{k}=\cos k_{x}-\cos k_{y}$. The sum is taken over the sublattice BZ (SBZ). The operators of the lower and upper SDW bands are related to the original $c$ operators by $a_{\mathbf{k} \sigma}=\alpha_{\mathbf{k}} c_{\mathbf{k} \sigma}+\sigma \beta_{\mathbf{k}} c_{\mathbf{k}+\mathbf{Q} \sigma}$ and $b_{\mathbf{k} \sigma}=-\sigma \beta_{\mathbf{k}} c_{\mathbf{k} \sigma}+\alpha_{\mathbf{k}} c_{\mathbf{k}+\mathbf{Q} \sigma}$, respectively. We set $\mathbf{Q}=(\pi, \pi)$ for the commensurate SDW state, $\alpha_{\mathbf{k}}^{2}=\frac{1}{2}\left[1-\left(\epsilon_{k} / \xi_{k}\right)\right]$, and $\beta_{\mathbf{k}}^{2}=\frac{1}{2}\left[1+\left(\epsilon_{k} / \xi_{k}\right)\right]$.

$H_{\mathrm{MF}}$ can be diagonalized separately for the lower and upper SDW bands. For the lower band, in addition to a constant, it becomes $\Sigma_{\mathbf{k}}-E_{k}\left(f_{1 \mathbf{k}}^{+} f_{1 \mathbf{k}}-f_{2 \mathbf{k}}^{+} f_{2 \mathbf{k}}\right) \quad$ where $f_{1 \mathbf{k}}=u_{k} a_{\mathbf{k} \uparrow}-v_{k} a_{-\mathbf{k} \downarrow}^{+}, f_{2 \mathbf{k}}=v_{k} a_{\mathbf{k} \uparrow}+u_{k} a_{-\mathbf{k} \downarrow}^{+}$, and

$$
E_{k}=\left(\xi_{k}^{2}+\Delta_{k}^{2}\right)^{1 / 2}=\left[\epsilon_{k}^{2}+\left(J m_{s}\right)^{2}+\Delta_{k}^{2}\right]^{1 / 2} .
$$

The coherence factors are $\left.u_{k}^{2}=\frac{1}{2}\left[1+\xi_{k} / E_{k}\right)\right]$, and $v_{k}^{2}=\frac{1}{2}$ $\left[1+\left(\xi_{k} / E_{k}\right)\right]$. In terms of the operators $a_{\mathbf{k} \sigma}^{+}$, the ground state has the familiar BCS form. Similarly, the upper SDW band also forms two bands with identical dispersion $\pm E_{k}$ as those of $f_{1 \mathbf{k}}$ and $f_{2 \mathbf{k}}$. If we had not chosen zero chemical potential for the half-filled case, there would be four nondegenerate bands as shown by Inaba et al. ${ }^{17}$

In the above approach, the local constraint of no doubly occupied sites is satisfied for the whole lattice on the average but it is not for each individual site. To obtain a more accurate quantitative result for the Hamiltonian $H$, we shall use the variational Monte Carlo (VMC) method that satisfies the constraint exactly. The ground state of $H_{\mathrm{MF}}$ is simply given by the product of two BCS-like wave functions, one for the lower SDW band and the other for the upper SDW band. In the presence of the constraint the wave function has the form

$$
\left|\Psi_{0}\right\rangle=P d\left[\sum_{k}\left(A_{k} a_{\mathbf{k} \uparrow}^{+} a_{-\mathbf{k} \downarrow}^{+}+B_{k} b_{\mathbf{k} \uparrow}^{+} b_{-\mathbf{k} \downarrow}^{+}\right)\right]^{N_{e} / 2}|0\rangle,
$$

where $N_{e}$ is the total number of electrons and coefficients $A_{k}=\left(E_{k}+\xi_{k}\right) / \Delta_{k}$ and $B_{k}=-\left(E_{k}-\xi_{k}\right) / \Delta_{k}$. The projection operator $P d$ enforces the constraint of no doubly occupied sites. In this wave function there are two variational parameters: $\Delta / \chi$ and $m_{s} / \chi$. In the absence of staggered order, $m_{s}$, this is exactly the same RVB wave function used by Gros. ${ }^{18}$ Without pairing this wave function describes the SDW state. $\left|\Psi_{0}\right\rangle$ is similar to the trial wave function used by Chen et al. ${ }^{14}$ but with a slightly lower energy. It has about $-0.332 J$ per bond which is within one percent of the best estimate of the ground-state energy of the Heisenberg model. The success of $\left|\Psi_{0}\right\rangle$ gives support to the mean-field theory that derives Eq. (4).

According to the mean-field Hamiltonian $H_{\mathrm{MF}}$ discussed above, creating a hole is to take away a quasiparticle $f_{1 \mathbf{k}}$ from the lower SDW band in the ground state, or the corresponding one in the upper SDW band. Hence the energy of such a state is just $-E_{g}+E_{k}$, where $-E_{g}$ is the ground-state energy at half filling. Interestingly, the quasiparticle disper-

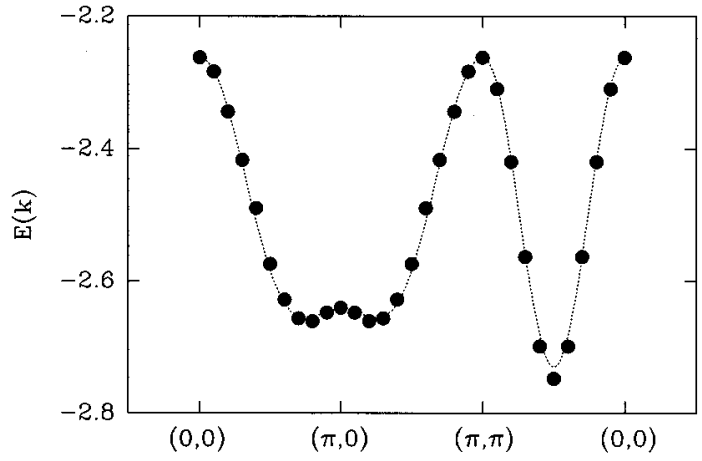

FIG. 1. Energy dispersion of a hole in the $t$ - $J$ model obtained by Liu and Manousakis (Ref. 7) with SCBA on an $20 \times 20$ cluster (solid circles), for $J=0.2$, and $t=1$. Dotted line is the fitted result of $E_{k}-E_{0}$ with $E_{0}=5.37, \chi=5.47, \Delta=2.27$, and $m_{s}=13.2$.

sion actually has the similar form as the dispersion of the hole obtained by SCBA. In Fig. 1 we show that the energy dispersion of a single hole obtained by Liu and Manousakis ${ }^{7}$ by using SCBA for a $20 \times 20$ lattice with $J=0.2$ can be fitted quite well by $E_{k}-E_{0}$ with parameters, $\chi=5.47, m_{s}=13.2$, and $\Delta=2.27$. $E_{0}=5.37$ is just a constant shift. In this paper, the energy unit is $t$. However, the value of $m_{s}$ is unphysically large. This is mainly due to our neglect of the constraint in deriving Eq. (3). The effect of antiferromagnetism is grossly overestimated. A more quantitative approach is to use the renormalized mean-field theory ${ }^{19}$ by taking into account the constraint a little bit more carefully. $m_{s}$ will be multiplied by a renormalization factor. Instead of pursuing this approach we shall use the variational method to calculate the dispersion numerically. Consequently, as shown below, more physically reasonable values of parameters are obtained. Here, the emphasis is that all the interesting features of the dispersion obtained by SCBA is quite consistent with the form of $E_{k}$.

To have a more accurate description of the single hole dispersion, we shall use the power method in addition to the VMC method. The power method ${ }^{20}$ which is essentially the same as the GFMC method, applies many powers of Hamiltonian to a trial wave function (TWF) to project out the ground state with the same symmetry as the TWF. To increase the convergence the method is modified by combining with the Lanczos method, we call it the power-Lanczos (PL) method. ${ }^{21,22}$

Following the mean-field theory discussed above, we can easily construct a variational wave function for a hole with momentum $\mathbf{k}$ and $S_{z}=1 / 2$. This function has $N_{e} / 2-1$ singlet pairs of electrons and a single unpaired electron with momentum $\mathbf{k}$ and $S_{z}=1 / 2$,

$$
\left|\Psi_{1}\right\rangle=P d c_{\mathbf{k} \uparrow}^{+}\left[\sum_{q}{ }^{\prime}\left(A_{q} a_{\mathbf{q} \uparrow}^{+} a_{-\mathbf{q} \downarrow}^{+}+B_{q} b_{\mathbf{q} \uparrow}^{+} b_{-\mathbf{q} \downarrow}^{+}\right)\right]^{N_{e} / 2-1}|0\rangle,
$$

where the prime on the summation symbol indicates that the momentum $\mathbf{k}$ is excluded from the sum if $\mathbf{k}$ is within the SBZ, otherwise, $\mathbf{k}-\mathbf{Q}$ is excluded. Notice that $\left|\Psi_{1}\right\rangle$ is essentially the same as $\widetilde{c_{-\mathbf{k} \downarrow}}\left|\Psi_{0}\right\rangle$. 


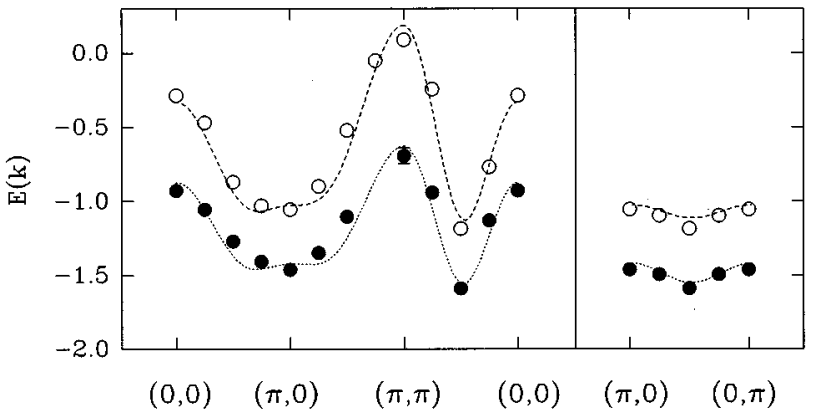

FIG. 2. Energy dispersion of a hole in the $t$ - $J$ model on an $8 \times 8$ cluster, for $J=0.3$, and $t=1$. Open circles are VMC results by using $\left|\Psi_{1}\right\rangle$, solid circles obtained by applying the PL method to this wave function. $E_{1 k}-E_{0}$ are plotted as the dotted lines. For the lower curve $\chi=6.92, \Delta=2.71, \quad m_{s}=18.84, \quad E_{0}=7.43$, and $t_{\text {eff }}=0.03$.

The energies obtained by the VMC method for an $8 \times 8$ cluster with $J=0.3$ are shown in Fig. 2 as the open circles. The variational parameters are $\Delta / \chi=0.3$ and $m_{s} / \chi=0.056$. Notice that the ground-state energy for the half-filled lattice $(-22.656 J)$ is subtracted from the data. The single-hole state has the lowest energy at $(\pi / 2, \pi / 2)$. We then apply the PL method to this TWF (Ref. 23) to project it onto the lowest energy state. Results are shown as the solid circles in Fig. 2.

The results represented by the solid circles in Fig. 2 are almost identical with that of Fig. 1. For both, the bandwidth is about $2.2 \mathrm{~J}$, the energy difference between states at $(\pi / 2, \pi / 2)$ and $(\pi, 0)$ is about $0.43 J$, and there is an EVHS around $(\pi, 0)$. However, there is one difference. In the SCBA, starting from the classical Néel state, the hole only hops between the same sublattice. The hole energy begins with $t^{2} / J$. States at $\mathbf{k}$ and $\mathbf{k}+\mathbf{Q}$ have the same energy. Our result shows an energy difference between $(\pi, \pi)$ and $(0,0)$ and a slight asymmetry between energies at $\mathbf{k}$ and $\mathbf{k}+\mathbf{Q}$. This is due to the nonvanishing hopping matrix element between the two sublattices which is related with the uniform bond order $\chi$. Kane et al. ${ }^{24}$ have pointed out this difference between a mean-field theory based on a Néel state or a RVB state.

Quantitatively, the difference between Figs. 1 and 2 can be accounted for by introducing a coherent nearest-neighbor hopping in addition to $E_{k}$ of Eq. (3), $E_{1 k}=E_{k}$ $-2 t_{\text {eff }}\left(\cos k_{x}+\cos k_{y}\right)$. Both the solid and open circles in Fig. 2 can be fitted very well by a functional form $E_{1 k}-E_{0}$, where $E_{0}$ is a constant. Although solid circles represent results much closer to the ground state than the results of TWF (open circles), the qualitative feature of the dispersion is essentially unchanged.

Much more accurate numerical results are obtained in the exact calculation for a 32-site cluster by Leung and Gooding $^{10,25}$ and in GFMC for a $12 \times 12$ cluster. $^{8}$ In Fig. 3, the 32-site result for $J=0.3$ is shown as open squares, solid circles are results from GFMC for $J=0.4$. Both dispersions can be fitted rather well by $E_{1 k}-E_{0}$ discussed above.

The results of our trial wave function shown in Fig. 2 agree fairly well with the more accurate results of Fig. 3. This certainly enhances our belief that the mean-field theory or the trial wave function $\left|\Psi_{1}\right\rangle$ has captured the essential

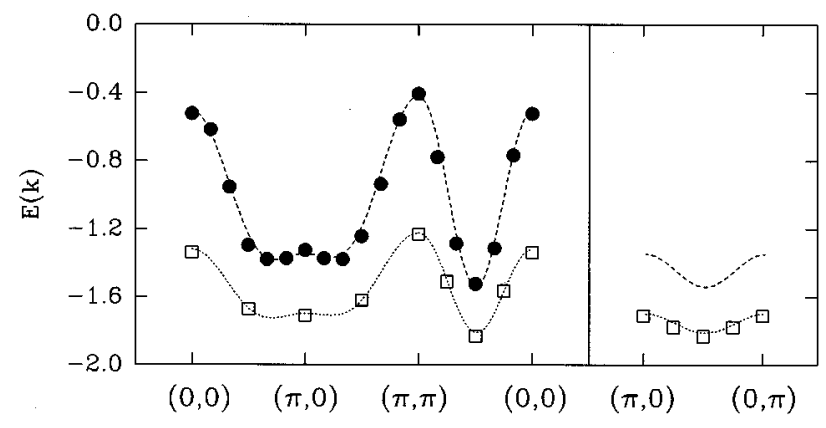

FIG. 3. Energy dispersion of a hole in the $t-J$ model on a 32-site cluster (open squares) obtained with exact diagonalization (Ref. 10) for $J=0.3$, and $t=1$. Solid circles are results of GFMC (Ref. 8) for a $12 \times 12$ cluster with $J=0.4$. Dotted lines are the fitted $E_{1 k}-E_{0}$ curves. For the upper curve (32 sites), $\chi=5.43, \Delta=2.43$, $m_{s}=17.6, E_{0}=7.09$, and $t_{\text {eff }}=0.012$. For the lower curve $(12 \times 12$ sites), $\chi=6.87, \Delta=2.83, m_{s}=18.4, E_{0}=8.9$, and $t_{\mathrm{eff}}=0.011$.

physics of the one-hole state. In particular, we notice that the asymmetrical energy dispersion between $\mathbf{k}$ and $\mathbf{k}+\mathbf{Q}$ are present in both 32 and 144 clusters. Although a careful and systematic investigation is necessary before we can firmly establish this asymmetry for larger clusters, this result clearly is in better agreement with our mean-field theory than the SCBA calculations. As explained above, even for small clusters, SCBA (Refs. 5-7) results still have the perfect antiferromagnetic symmetry.

Another interesting result is the observation of the flat band region, which is the so-called EVHS, ${ }^{9}$ near $(\pi, 0)$ in Figs. 2 and 3. Examining $E_{k}$ or $E_{1 k}$ shows that EVHS is due to the presence of $\Delta$ or $d$-RVB order parameter and it is not a consequence of long-range antiferromagnetic ordering. The curvature of the dispersion at $(\pi, 0)$ depends on the ratio $\Delta / \chi$. The $d$-RVB order parameter has the largest band gap near $(\pi, 0)$. This band gap will produce large density of states which is reflected by this EVHS.

In our calculation of the lowest energy state for each wave vector $\mathrm{k}$, it is not guaranteed that the state we obtained has a finite spectral weight when a hole is produced from the halffilled ground state. It is therefore necessary to examine the spectral weight $Z_{\mathbf{k}}$ defined as

$$
Z_{\mathbf{k}}=\left|\left\langle\Psi_{\mathbf{k}}\left|c_{\mathbf{k}, \sigma}\right| \Phi\right\rangle\right|^{2}
$$

where $|\Phi\rangle$ is the normalized exact ground state at half filling and $\left|\Psi_{\mathbf{k}}\right\rangle$ is the lowest energy state at momentum $\mathbf{k}$ in the presence of one hole. Here we use VMC and PL method to calculate $Z_{\mathbf{k}}$. The trial functions are $\left|\Psi_{0}\right\rangle$ of Eq. (4) and $\left|\Psi_{1}\right\rangle$ used to obtain Fig. 2. The results for 64 sites are plotted in Fig. 4. Open circles are VMC results and solid circles are results of first order PL. ${ }^{21,23}$ We have also included the exact result of 32 sites $^{10}$ as the open squares. For most wave vectors our results have roughly the right magnitude except near $\mathbf{k}=(0,0)$. Clearly, we have not yet obtained the exact results for 64 sites and more higher order power method calculations are needed. However, the result is enough to see the asymmetry between $\mathbf{k}$ and $\mathbf{k}+\mathbf{Q}$, which is absent in the SCBA. 


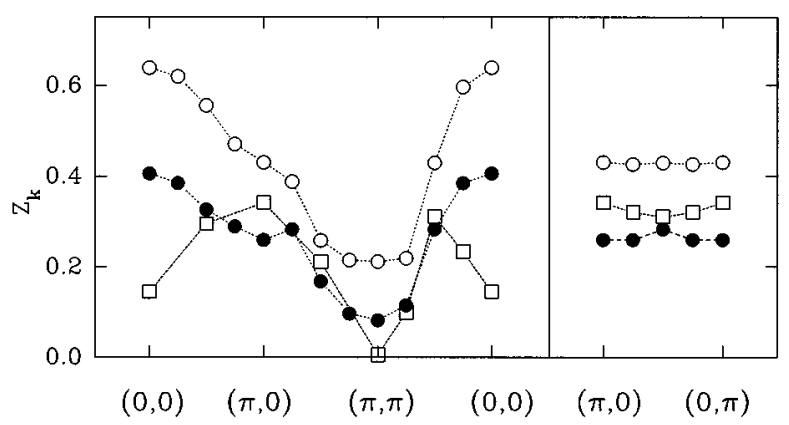

FIG. 4. Spectral weight $Z_{\mathbf{k}}$ as a function of $\mathbf{k}$ for the $8 \times 8$ cluster used in Fig. 2. Open circles are VMC results and solid circles are by PL1. The exact results of 32 sites are shown as open squares.

So far we have assumed that the hole only has the nearestneighbor hopping matrix element. To explain the ARPES (Ref. 3) result, there has been effort ${ }^{11,12}$ to generalize the model by including the next-nearest-neighbor hopping, $t^{\prime}$, and next-next-nearest-neighbor hopping, $t^{\prime \prime}$. Fairly complicated results obtained in these cases can be simply understood in our mean-field theory. The basic dispersion is determined by the spin interaction. The additional contribution by $t, t^{\prime}$, and $t^{\prime \prime}$ is the same as that of the ideal gas except with a renormalized magnitude. Hence for the $t-t^{\prime}-t^{\prime \prime}-J$ model, the one-hole dispersion has the form, $E_{2 k}=E_{1 k}$ $-4 t_{\text {eff }}^{\prime} \cos k_{x} \cos k_{y}-2 t_{\text {eff }}^{\prime \prime}\left[\cos \left(2 k_{x}\right)+\cos \left(2 k_{y}\right)\right]$. This is verified in Fig. 5.

In Fig. 5 the first-order Lanczos result ${ }^{23}$ of using $\left|\Psi_{1}\right\rangle$ for the $t-t^{\prime}-t^{\prime \prime}-J$ model with $J=0.3$, and $t^{\prime}=-0.3, t^{\prime \prime}=0$ is shown as the circles, $t^{\prime}=-0.3$ and $t^{\prime \prime}=0.2$ as the squares. We have shifted the two sets of data so they match at $\mathbf{k}=(\pi / 2, \pi / 2)$. The lower dotted curve shows the fitted result by $E_{2 k}$ with $t_{\text {eff }}^{\prime}=0.092$ and $t_{\text {eff }}^{\prime \prime}=0$. The squares are fitted with $t_{\text {eff }}^{\prime}=0.065$ and $t_{\text {eff }}^{\prime \prime}=-0.055$. All other fitted parameters are the same as those used in Fig. 2.

Notice that $t_{\text {eff }}^{\prime}$ has the opposite sign of $t^{\prime}$. This can be easily understood in terms of the $d$-RVB property of the TWF. When the hole hops from site $i_{x}, i_{y}$ to site $i_{x}+1, i_{y}+1$, it rotates a nearest-neighbor RVB bond in the

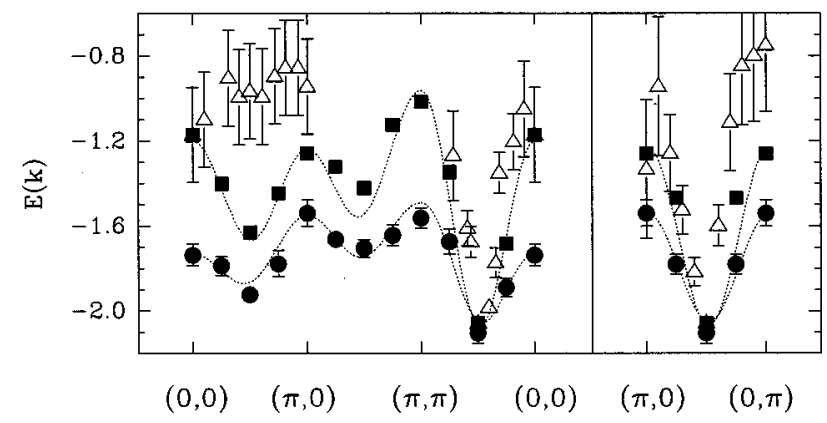

FIG. 5. Energy dispersion of a hole in the $t-t^{\prime}-t^{\prime \prime}-J$ model on an $8 \times 8$ cluster, for $J=0.3, t^{\prime}=-0.3$, and $t=1$. Circles are for $t^{\prime \prime}=0$ and squares for $t^{\prime \prime}=0.2$. Both results are obtained by using first order PL method on $\left|\Psi_{1}\right\rangle$ (Ref. 23). Triangles are the rescaled ARPES data (Ref. 3). $E_{2 k}-E_{0}$ are plotted as the dotted lines. The parameters are the same as those in Fig. 2, except $t_{\text {eff }}^{\prime}=0.092$ for the lower curve, and for the upper curve $t_{\mathrm{eff}}^{\prime}=0.065$ and $t_{\mathrm{eff}}^{\prime \prime}=-0.055$. $y$ direction to $x$ direction. Due to the $d$ symmetry of the singlet bond, such a rotation produces a negative sign in the hopping matrix element. Similarly for $t^{\prime \prime}$, a nearest-neighbor RVB bond is reversed, and $t_{\text {eff }}^{\prime \prime}$ has the opposite sign.

From Fig. 5 or $E_{2 k}$, we can see that the bandwidth, which is the energy difference between $\mathbf{k}=(0,0)$ and $(\pi / 2, \pi / 2)$, would be reduced if $t^{\prime}$ is negative and $t^{\prime \prime}=0$. But for $t^{\prime \prime}$ to be positive and of the similar magnitude as $t^{\prime}$, the bandwidth is about the same as the $t-J$ model. While for most region of the BZ the effect of $t^{\prime}$ and $t^{\prime \prime}$ cancels each other, but near $(\pi, 0)$ they add up. Hence the most significant change between Figs. 2 and 5 is the energy increase for the state at $(\pi, 0)$. This increase is necessary to make the theory in better agreement with the ARPES data by Wells et al. ${ }^{3}$ The ARPES data are the open triangles in Fig. 5. We have rescaled the data so that the energies at $(0,0)$ and $(\pi 2, \pi / 2)$ match that of the squares in Fig. 5. Notice that we have not chosen $t^{\prime}$ or $t^{\prime \prime}$ in order to match the experiments. Even if we had used the matched $t^{\prime}$ or $t^{\prime \prime}$, the discrepancy between theory and experiment along $(1,0)$ direction will not be removed. This can be easily understood by examining $E_{2 k}$. The energy at $(\pi, 0)$ may be shifted by $t^{\prime}$ and $t^{\prime \prime}$, but not at $(\pi / 2,0)$. Unlike other well-known high-temperature superconductors, $\mathrm{Sr}_{2} \mathrm{CuO}_{2} \mathrm{Cl}_{2}$ is known to be fairly difficult to be doped into a superconductor. We note that since $(\pi, 0)$ is exactly at the EVHS region with a large density of states, some subtle difference between materials may become important. The experimental analysis is further complicated by the recent observation of the $d$-wave-like gap structure ${ }^{26,27}$ near $(\pi, 0)$. More accurate experimental results for $\mathrm{Sr}_{2} \mathrm{CuO}_{2} \mathrm{Cl}_{2}$ and other hightemperature superconductors are needed to clarify the discrepancy between theory and experiment.

In summary, by using a mean-field theory that takes into account both staggered magnetization and $d$-RVB singlet we have derived the energy dispersion of a single hole in the $t-J$ and $t-t^{\prime}-t^{\prime \prime}-J$ models. Numerical results obtained from exact diagonalization, GFMC method and VMC are all in good agreement with the dispersion relation. In this theory, occurrence of the EVHS near $(\pi, 0)$ is due to the presence of $d$-RVB order parameter. The dispersion does not have the exact sublattice symmetry observed in the Néel state in which $\mathbf{k}$ and $\mathbf{k}+\mathbf{Q}$ are degenerate. This particular feature agrees with results obtained by exact diagonalization and GFMC method and disagrees with the SCBA. Simple arguments have been provided to account for this difference.

It should be pointed out that there are other quite successful variational studies ${ }^{28}$ about the single-hole dispersion. Although the wave functions were constructed in different ways, it was recognized that the spin-flip terms are essential in obtaining the right physics. The energy due to the spin-flip term is exactly what the RVB order parameters are designed for. We believe that one of the main reasons why so many different calculations mentioned in this paper all have obtained similar results is that they all have taken into account the dominant spin-flip effect. The RVB theory is the easiest way to take into account of this effect right from the beginning. It also gives a more intuitively simple interpretation of the dispersion and EVHS.

The EVHS has been observed in many high-temperature superconductors. There is not yet a convincing theoretical reason to explain it. Although the theory presented here is only valid in the presence of a single hole, we can make a 
few general comments about the underdoped region. In many earlier numerical studies the $d$-RVB state has been known to give a good account of the ground state of the $t-J$ model. The $d$-RVB order parameter would produce a gap and a large density of state or EVHS at $(\pi, 0)$. Such a gap may provide a natural explanation for the spin gap and the gap observed in ARPES. ${ }^{26,27}$ Theoretical work is now in progress to address these issues.
We wish to thank Y.C. Chen, P.W. Leung, and Yu Lu for many useful discussions. Part of the research was conducted using the resources of the Cornell Theory Center which receives major funding from NSF and NY state with additional support from ARPA, NIH, IBM, and members of the Corporate Research Institute. Part of the computations were performed at the National Center for High Performance Computing in Taiwan. We are grateful for their support.
${ }^{1}$ D.S. Dessau et al., Phys. Rev. Lett. 71, 2781 (1993).

${ }^{2}$ K. Gofron et al., Phys. Rev. Lett. 73, 3302 (1994).

${ }^{3}$ B.O. Wells et al., Phys. Rev. Lett. 74, 964 (1995).

${ }^{4}$ A very good review by $\mathrm{Yu} \mathrm{Lu}$ et al., Chin. J. Phys. 31, 579 (1993).

${ }^{5}$ F. Marsiglio et al., Phys. Rev. B 43, 10882 (1991).

${ }^{6}$ G. Martinez and P. Horsch, Phys. Rev. B 44, 317 (1991).

${ }^{7}$ Z. Liu and E. Manousakis, Phys. Rev. B 45, 2425 (1992).

${ }^{8}$ E. Dagotto et al., Phys. Rev. Lett. 73, 728 (1994).

${ }^{9}$ E. Dagotto et al., Phys. Rev. Lett. 74, 310 (1995).

${ }^{10}$ P.W. Leung and R.J. Gooding, Phys. Rev. B 52, 15711 (1995).

${ }^{11}$ A. Nazarenko et al., Phys. Rev. B 51, 8676 (1995).

${ }^{12}$ T. Xiang and J.M. Wheatley, Phys. Rev. B 54, R12 653 (1996).

${ }^{13}$ T.K. Lee and S. Feng, Phys. Rev. B 38, 11809 (1988).

${ }^{14}$ G.J. Chen et al., Phys. Rev. B 42, 2662 (1990).

${ }^{15}$ T. Giamarchi and C. Lhuillier, Phys. Rev. B 43, 12943 (1991).

${ }^{16}$ P.W. Anderson, Science 235, 1196 (1987).

${ }^{17} \mathrm{M}$. Inaba et al. (unpublished).

${ }^{18}$ C. Gros, Phys. Rev. B 38, 931 (1988).
${ }^{19}$ F.C. Zhang et al., Supercond. Sci. Technol. 1, 36 (1988).

${ }^{20}$ Y.C. Chen and T.K. Lee, Z. Phys. B 95, 5 (1994).

${ }^{21}$ Y.C. Chen and T.K. Lee, Phys. Rev. B 51, 6723 (1995).

${ }^{22}$ E.S. Heeb and T.M. Rice, Z. Phys. B 90, 73 (1993).

${ }^{23}$ The solid circles shown in Figs. 2 and 5 used the first order Lanczos to improve the TWF, this is called PL1, and then apply two powers of the Hamiltonian. The solid circles in Fig. 4 and squares in Fig. 5 are PL1 without extra powers.

${ }^{24}$ C.L. Kane et al., Phys. Rev. B 39, 6880 (1989).

${ }^{25}$ There are typos in Fig. 3 and Table I in Ref. 10. The energy values at $(\pi, \pi / 2)$ and $(\pi / 2,0)$ should be exchanged. The result is then consistent with the term, $2 t_{\text {eff }}\left(\cos k_{x}+\cos k_{y}\right)$, in $E_{1 k}$. The energy inside the SBZ should always be lower than the value at the corresponding $\mathbf{k}$ outside the SBZ.

${ }^{26}$ A.G. Loeser et al., Science 273, 325 (1996).

${ }^{27}$ H. Ding et al., Nature 382, 51 (1996).

${ }^{28}$ M. Boninsegni and E. Manousakis, Phys. Rev. B 43, 10353 (1991); 45, 4877 (1992). 